DOI: https://doi.org/10.46296/yc.v5i9edespsep.0102

\title{
COMPORTAMIENTO Y LEALTAD DEL CONSUMIDOR SOBRE LOS ESTABLECIMIENTOS GASTRONÓMICOS DE QUITO POR EFECTO DEL COVID-19
}

\section{LOYALTY AND CONSUMER BEHAVIOR RELATED TO FOOD BUSINESSES IN QUITO DUE TO THE EFFECT OF COVID-19}

\author{
Salazar-Duque Diego ${ }^{\text {; }}$ Burbano Camila ${ }^{2}$; Portugal Carolina ${ }^{3}$; Guamialama Jaime ${ }^{4}$ \\ ${ }^{1}$ Universidad UTE; CiTUR ECUADOR. Quito, Ecuador. Correo: \\ diegoa.salazar@ute.edu.ec. ORCID ID: https://orcid.org/0000-0003-2111-9134 \\ 2 Investigadora independiente. Quito, Ecuador. Correo: \\ camilaburbanoargoti@gmail.com. ORCID ID: https://orcid.org/0000-0003-3515-6673
}

${ }^{3}$ Universidad UTE. Quito, Ecuador. Correo: elizabeth.portugal@ute.edu.ec. ORCID ID: https://orcid.org/0000-0002-8398-4839
${ }^{4}$ Universidad UTE. Quito, Ecuador. Correo: jguamialama@ute.edu.ec. ORCID ID: https://orcid.org/0000-0002-9591-1258

\begin{abstract}
Resumen
La presente investigación analiza los factores que influyen en el comportamiento y la lealtad del consumidor sobre los establecimientos gastronómico por el impacto que ha generado en el individuo el COVID-19. Para ello, se toma como base aquellos estímulos del entorno y del marketing mix que influyen en la lealtad del consumidor, así como el proceso de compra. La metodología considerada para este estudio es de carácter cuantitativa; para ello se levantó la opinión pública de 600 personas; consecuentemente se consideró un análisis de fiabilidad basado en el cálculo del coeficiente alfa de Cronbach y un análisis factorial exploratorio mediante el cálculo de los coeficientes de correlación argumentada por el test estadístico de Bartlett y el $\mathrm{KMO}$. Como resultado principal se puede observar que aquellos estímulos que provienen del entorno como aquellos que son generados por las empresas a partir del marketing mix, influyen significativamente en la lealtad hacia una empresa del sector de alimentos y bebidas. Se concluye que, los estímulos del entorno, los estímulos del marketing mix y el proceso de compra influyen significativamente en la lealtad en el sector de alimentos y bebidas.
\end{abstract}

Palabras claves: comportamiento del consumidor, lealtad, marketing mix, proceso de compra.

\begin{abstract}
This research analyzes the factors that influence consumer behavior and loyalty in food industry due to the impact of COVID-19 in individuals. Those stimuli from the environment and marketing mix, consumer loyalty, and purchase process have been taken as a basis. The methodology considered for this study is quantitative. Public opinion from 600 people were analyzed. Consequently, a reliability analysis was considered on the calculation of Cronbach's alpha coefficient. An exploratory factor analysis was made up by calculating the correlation coefficients based on the Bartlett and KMO statistical tests. As main result, it can be observed that those stimuli that come from the environment the ones which are generated by companies from marketing mix directly influence in loyalty to a company in food industry. It is concluded that environmental stimuli, marketing mix stimuli, and purchase process persuade loyalty in food stablishments.
\end{abstract}

Keywords: customer behavior, loyalty, marketing mix, purchasing process.

Información del manuscrito:

Fecha de recepción: 14 de diciembre de 2020.

Fecha de aceptación: 09 de septiembre de 2021.

Fecha de publicación: 17 de septiembre de 2021. 


\section{Introducción}

La lealtad es un comportamiento emocional y de confianza que se desarrolla entre el consumidor y la empresa a largo plazo. Este tipo de conducta se concibe por el nivel de interés que tiene una persona, al satisfacer una misma necesidad durante la compra reiterada de un mismo producto, una marca, o la frecuencia periódica en un mismo establecimiento. De acuerdo a Rivas e Idefonso (2013), este tipo de reacción se genera en el consumidor por efecto del reconocimiento, frecuencia de compra, e insistencia de recompra, los cuales se generan por la eliminación de las diversas alternativas existentes en el mercado en favor de su preferencia final.

Ante este escenario, todos aquellos esfuerzos comerciales que considere una organización, permitirán alcanzar una lealtad, atraer nuevos clientes e incrementar la rentabilidad (López et al, 2018). Es indispensable que las metas $u$ objetivos de una organización, sean controlados paulatinamente a partir de varios indicadores clave de rendimiento $(\mathrm{KPI})$ como la cuota de mercado, nivel de satisfacción del consumidor, entre otros (dos Anjos et al, 2019; Candela, 2019). Según Munuera y Rodríguez (2016), las estrategias empresariales orientadas al mercado deben estar encaminadas a tener una ventaja competitiva, que le permita sostenerse en el tiempo considerando los recursos, la capacidad de la empresa, y el entorno en el cual opera.

Ante esta realidad, el modelo de comportamiento generado por el consumidor y la decisión de compra se ha efectuado mecánicamente durante muchos años, proporcionados por las distintas empresas para cumplir diversas necesidades, gustos, preferencias según la accesibilidad que tiene cada individuo sobre aquellos productos o servicios buscados. No obstante, tras el confinamiento generado por efecto del COVID-19 a nivel mundial (Sohrabi et al, 2020), la preocupación 0 miedo del ser humano ante un posible contagio por consecuencia de comprar un producto o servicio que haya tenido algún contacto con el virus (Johnson et al, 2020; Huarcaya-Victoria, 2020), y las nuevas normativas para reactivar los negocios, podrían 
cambiar este comportamiento de compra y; por ende, influir en la lealtad hacia la marca. Hecho que se observa drásticamente por el incremento de compras a domicilio por concepto del delivery, el uso frecuente de aplicaciones digitales que se relacionan con este tipo de actividad comercial, la necesidad de nuevos empaques para facilitar el transporte de los productos, y, la información mediática que desarrolla un negocio de alimentos y bebidas para que se efectúe de forma efectiva este proceso administrativo y social.

Por consiguiente; y con el fin de identificar qué aspectos podrían afectar o favorecer la lealtad a futuro entre la empresa y el consumidor, por efecto de este nuevo virus, esta investigación tiene como principal objetivo, analizar si los factores exógenos o endógenos que influyen en el comportamiento del consumidor, también influyen en la lealtad de un individuo sobre los establecimientos gastronómicos del sector de alimentos y bebidas por el impacto que ha generado en esta persona el COVID-19. Con ello se podrá, a nivel empresarial, generar cambios estructurales y adaptar a sus negocios a la nueva realidad mediante cambios drásticos, si quiere sobrevivir.

\section{Teoría y conceptos}

\subsection{El sector de alimentos $y$ bebidas}

Previo el advenimiento de la pandemia del COVID - 19, el sector de alimentos y bebidas tenía el potencial para combinar sus recursos (López, 2003) con el fin de cubrir las necesidades gastronómicas y culinarias de acuerdo a ciertas tendencias, tradiciones, costumbres y cambios que surgen en las sociedades con el paso del tiempo; con lo que se vinculaban de manera directa con las actividades turísticas de una determinada región (Gomes et al, 2013), las cuales generaban una contribución importante dentro de la localidad y, consecuentemente en la región (Gallegos, 2002; Salazar, 2016). En este sentido, la conducta de la oferta se ve relacionada con la conducta de la demanda (Lamb, 2019) la que, a su vez, se veía directamente influenciada por el comportamiento del consumidor y sus hábitos gastronómicos (Rivas e 
Ildefonso, 2013; Salazar, 2016), mismas que dependen hasta la actualidad de las condiciones geográficas en las que se encuentren.

Por consecuencia del COVID-19, el sector de alimentos y bebidas ha sido uno de los más golpeados por esta pandemia (CEPAL, 2020). En este sentido, los establecimientos dedicados al servicio de alimentos y bebidas, lugares de concurrencia masiva de personas que acudían no solo para alimentarse, sino también para compartir tiempo de calidad con su entorno, consideraron el distanciamiento físico entre empresa y cliente (Organización Mundial de la Salud, 2020); de igual forma, la mayoría contempló ajustes en su nómina con el fin de asegurar la sostenibilidad del negocio; también potenció sus actividades a nivel tecnológico o e-commerce de la noche a la mañana mediante plataformas para entregar su producto al cliente, o mediante negociaciones con plataformas con experiencia en entrega para este tipo de servicio al cliente para generar sus ventas por concepto de delivery, que garanticen altos estándares de higiene y manipulación de productos evitando de esta manera el contacto directo y una posible propagación del virus.

En la actualidad, el COVID-19 ha dejado a la población a nivel mundial muy golpeada (CEPAL, 2020). Para el sector de alimentos y bebidas, regresar a la "nueva normalidad", genera una oportunidad para transformar e innovar procesos y procedimientos anclados en protocolos de bioseguridad, que cumplan con las disposiciones de las autoridades locales para la reapertura controlada de este sector como una correcta señalética, distancia entre mesas, uso constante de elementos de desinfección en equipos, personal y clientes, traslado de alimentos con BPM para servicio a domicilio, entre otras normativas que serán ahora parte fundamental para una nueva operación (Ministerio de Turismo, 2020).

\subsection{La lealtad del consumidor}

La lealtad del consumidor es un comportamiento afectivo que se genera de una persona hacia un lugar, un producto, un servicio o una marca. Es decir, un sentimiento de agrado o desagrado hacia el objeto 
(McGuire, 1968 referido por Ubillos et al, 2005).

A nivel empresarial, este comportamiento puede influir en la persona por efecto de una constante innovación, (Pappu y Genevieve, 2016), credibilidad, y percepción (Alguacil et al, 2016; Yang, 2020) percibida por la calidad que posee un producto 0 servicio. Un establecimiento de alimentos 0 bebidas deberá brindar satisfacción, confianza, apego y compromiso (Lombart y Didier, 2016; Sanz et al, 2018) sobre sus productos y servicios con la intención de que un cliente vuelva a futuro y permita generar lealtad. Para alcanzar esta postura de lealtad, se la deberá construir sobre tres posibles escenarios: 1) sobre un cliente que es propenso a ser leal por tendencia; 2) sobre un cliente quien manifiesta fidelidad por un lugar concreto con intención de compra por recomendación boca - oído; y 3) sobre una fidelidad actitudinal por la buena experiencia recibida por el trato de los empleados (Moliner y Berenguer, 2011; García y Fabero, 2016).

Bajo este preámbulo, la lealtad del consumidor con la empresa será influenciada directa e indirectamente por una Responsabilidad Social Corporativa (RSC) a través de la admiración y reputación percibida, (Castro-González y Bande, 2016) la cual se fortalecerá posteriormente con la compra. Ante este modelo, la posición que sostiene el marketing relacional sobre la lealtad, es que las estrategias que se consideren para este comportamiento, tanto con los nuevos clientes como con los antiguos clientes (Arguello et al, 2017; Salazar et al, 2016).

Para Kotler y Armstron (2012), los diversos estímulos externos e internos que intervienen en el consumidor ante una decisión de compra (marketing mix y los factores del entorno), y aquellas características propias que definen a un individuo como comprador, (Izurieta, 2015) inciden en el comportamiento del consumidor y pueden estar vinculadas con la actitud posterior a la compra (Ubillos et al, 2005; Zeithaml et al, 2009; Kotler y Armstrong, 2012) que se ejercen como una predisposición favorable a un compromiso de compra repetida como paso previos a la acción de compra (Oliver, 1999 referido por Forgas et al, 2011). 
Según Kotler y Armstrong (2012), existen ciertos factores culturales, sociales, conductuales 0 psicológicos, que caracterizan a un individuo ante una decisión de compra. Es decir, a raíz de esta pandemia, por ejemplo, se contempla una disonancia cognitiva entre los consumidores a nivel psicológico; una tensión o presión que siente el cliente después de una compra de un producto. Este tipo de conducta por parte del consumidor, en el sector de la hospitalidad, está relacionado directamente por un modelo tridimensional compuesto por tres componentes: a) cognitivos (creencias y opiniones que un individuo posee sobre un producto $o$ servicio donde se incluye la información),

b) afectivos (sentimientos de agrado 0 desagrado hacia un producto 0 servicio), y c) conativos-conductual (tendencias, disposiciones $\mathrm{O}$ intenciones de compra ante un producto o servicio) (McGuire, 1968, 1985; Breckler, 1984; Judd y Johnson, 1984; Chaiken y Stangor, 1987 referidos por Ubillos et al, 2005) al que se le puede adjuntar la d) de acción (proceso de compra de un producto o servicio); componentes que se definen como una promesa de comportamiento relativo al producto o servicio, mismo que conlleva la probabilidad de compra futura, o a la posibilidad de que el cliente cambie a otra marca $o$ proveedor del servicio (Bigné y Andreu, 2004; Arguello et al, 2017; Sanz et al, 2018).

En este sentido, para que se genere un efecto de lealtad en el consumidor, entre los factores que se conectan entre sí, y que influyen en la elección de un producto, servicio o marca se destacan: 1) aquellos estímulos exógenos (entorno) que influyen positiva o negativamente en su decisión final de compra; 2) la auto información, consciente $\mathrm{O}$ inconsciente, de los distintos productos, servicios, marcas o establecimientos que puedan participar en el mercado como opciones de elección (oferta/marketing mix), relacionados con la expectativa, calidad y el valor percibido de cada uno de ellos a través de elementos claves como: las personas, los procesos y la evidencia física, más aquellos componentes tradicionales como: el producto, el precio, la plaza y la promoción (Booms y Bitner, 1981; Zeithaml et al, 2009; Uddin, 2016; 
Saidani y Sudiarditha, 2019; Hung Do y Anh Vu, 2020); y 3) la auto evaluación percibida como cliente para el proceso de compra que posteriormente se reflejarán mediante un nivel de satisfacción (Fornell, 1996; Saidani y Sudiarditha, 2019; Yang, 2020) a través de su perspectiva actitudinal (Moliner y Berenguer, 2011)

Relación entre los estímulos del entorno del consumidor y la lealtad

El comportamiento del consumidor es una actitud que refleja cada individuo por consecuencia de características exógenas y endógenas que influyen en el consumidor. Con base en la postura presentada por Kotler y Armstrong (2012), entre los estímulos externos del entorno se destacan factores como: económicos, tecnológicos, políticos y culturales. No obstante, existen otros elementos que no sólo interfieren en la decisión de compra del cliente, sino que también en el desarrollo de una empresa: demográficos o socio-culturales, medioambientes o legales (Osorio et al, 2016; de Almeida et al, 2015; Izurieta, 2015; Salazar, 2016; Macas et al, 2018) y que pueden influir en la lealtad. Por su parte, Kotler y Keller (2012), resaltan características del consumidor como: personales, compuestos por: la edad y la etapa del ciclo de vida, la ocupación y las circunstancias económicas, la personalidad y el concepto personal, el estilo de vida y los valores; sociales, por: grupos de referencia, familia, roles y estatus sociales; y culturales, por: la cultura del individuo, subcultura y clase social. La hipótesis planteada será:

Hi1: Los estímulos del entorno influyen significativamente en la lealtad del consumidor.

Relación entre los estímulos del marketing mix en el consumidor $y$ la lealtad

Históricamente los elementos del marketing mix han sido considerados como los pilares fundamentales de toda disciplina que se relacione con el Marketing para influir significativa en el proceso de compra. Entre los elementos tradicionales (4 P's) se destaca el producto, el precio, la plaza y promoción. No obstante, a través del tiempo han ido evolucionando, incorporando o adaptando otros elementos según la orientación en la que se desee 
aplicar (7 P's, 8 P's, etc) (Bitner, 1991; Goi, 2009; Zeithaml et al, 2009; Hoffman y Bateson, 2012; Mahajan y Golahit, 2017; Sisodiya, 2018; Wu y Li, 2018; Lamb, 2019; Xie, 2020); siendo aspectos claves para generar valor a un producto 0 servicio, y que éstos a su vez puedan ser percibidos por un cliente, quien busca cierto valor de la empresa percibidos como un consumidor, costo, conveniencia y comunicación (4 C's) Lauterborn (1990) referido por Draskovic y Valjak (2012); y Panigrahi et al (2018).

Ante este punto de vista, la decisión de compra sobre el producto 0 servicio en las que puede evaluar un consumidor se generan sobre la base de las 4 P's del Marketing, las cuales están estrechamente relacionadas con las 4 C's del Marketing. Es decir; para generar un buen producto, será necesario estudiar los deseos o necesidades que tiene un consumidor (Draskovic y Valjak, 2012) sobre qué cartera de productos o servicios debería tener una empresa de alimentos y bebidas, la diferenciación, diseño o características de los productos 0 servicios que necesitan, gustan 0 desean, el empaque más adecuado para transportar alimentos a domicilio, o su percepción ante la marca (Serra, 2012; Kotler y Armstrong, 2012; Lamb, 2019).

A nivel de precio, será necesario estimar el costo (Draskovic y Valjak, 2012) que le representa a este cliente un producto o servicio sobre el valor por el que debe pagar como consumidor sobre un bien tangible 0 intangible y las formas de pago que se generen sobre éstos (Lamb, 2019).

Por otro lado, es necesario delimitar la importancia que tiene la conveniencia (Draskovic y Valjak, 2012) para un consumidor a partir de la forma o manera que prefiere adquirir un producto o servicio; siendo necesario evaluar como empresa los canales de distribución, la ubicación y horario de atención, el ambiente 0 diseño del establecimiento y los procesos de venta; los cuales son coadyuvados como criterios contemplados desde la plaza o distribución (Lamb, 2019).

Finalmente será necesario valorar la interacción que debe existir entre la empresa-empleados y los clientes a través de la promoción, mediante una correcta comunicación 
(Draskovic y Valjak, 2012) reflejada por medio de una correcta: publicidad o marketing directo, venta personal, promociones de venta, social media y relaciones públicas (interna y externa a la empresa) (Serra, 2012; Lamb, 2019),

De acuerdo con Kotler y Armstrong, (2012) estos componentes (4 P's) son estímulos exógenos que influyen en el comportamiento del consumidor. Según Setiawan (2019); Xie (2020) y Yelkur (2020), existe una correlación positiva entre la mezcla de marketing y la satisfacción del consumidor. De acuerdo a Kotler y Armstrong, (2012), el producto está compuesto por: la variedad de productos, calidad, diseño, características, marca, envasado, tamaños, servicios, garantías y devoluciones; el precio por: el precio de lista (tarifa), descuentos, incentivos, periodo de pago, condiciones de crédito; la promoción: promoción de ventas publicidad, fuerza de ventas, relaciones públicas, marketing directo, y la plaza de: canales, cobertura, surtidos, ubicaciones, inventario y transporte. Se supone por lo tanto que los estímulos de marketing ejercen una influencia directa sobre la lealtad. La hipótesis propuesta será la siguiente:

Hi2: Los estímulos de marketing mix influyen significativamente en la lealtad del consumidor.

Relación entre el proceso de compra del consumidor y la lealtad

Según Peñalosa et al (2018), es importante que el cumplimiento, privacidad y satisfacción que genere una organización sean factores que influyan positivamente en la lealtad del consumidor de servicios. Para Kotler y Armstrong (2012), este individuo debe pasar preliminarmente por varias etapas, actividades 0 acciones durante un proceso de compra antes de generar una lealtad (reconocimiento de las necesidades, búsqueda de información, evaluación de alternativas, decisión de compra, y el comportamiento posterior a la compra). La experiencia en su última etapa como consumidor acorde al desempeño percibido por parte de la marca, producto o servicio, y puede afectar de forma positiva o negativa a la lealtad (Moliner y Berenguer, 2011). La propuesta de hipótesis considerada es: 
Hi3: El proceso de compra influye significativamente en la lealtad

La figura 1 muestra los componentes del comportamiento del consumidor que influyen positivamente en la formación de la lealtad considerados para esta investigación.

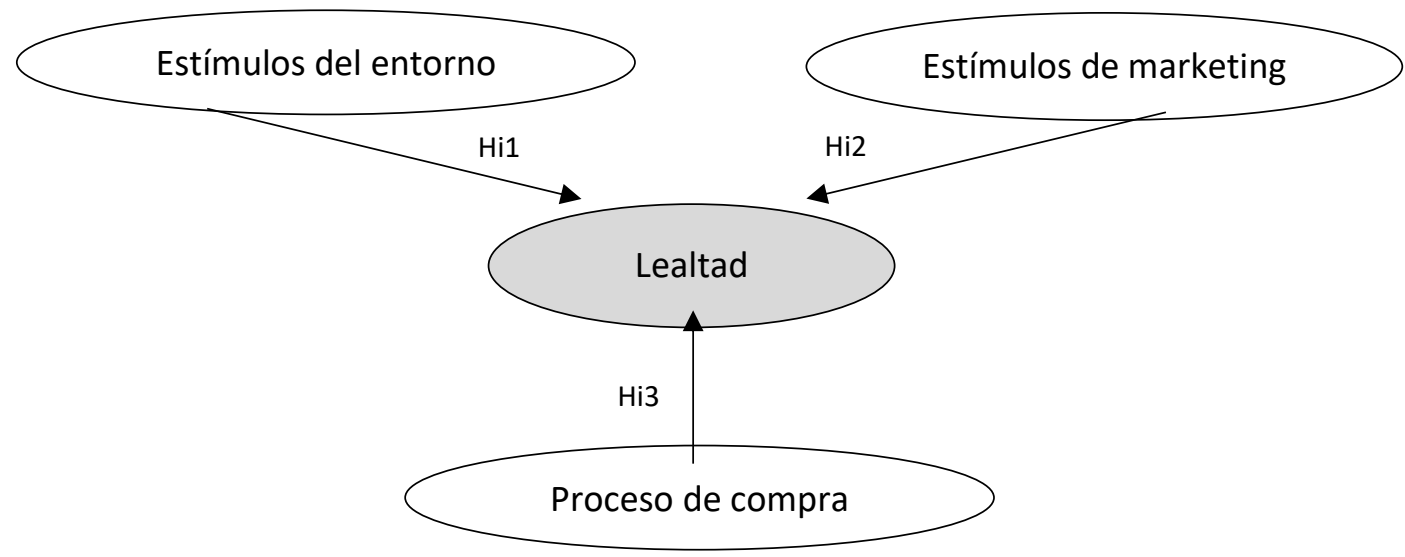

Figura 1. Componentes del comportamiento del consumidor en la formación de la lealtad.

\section{Metodología}

\subsection{Proceso metodológico}

La metodología considerada para este estudio se genera mediante una investigación cuantitativa, la cual busca conocer, explicar y predecir la relación entre variables identificadas en este manuscrito de forma imparcial sobre los resultados que se obtengan (Luque, 2017). Para este proceso, se consideró levantar la información con ayuda de un cuestionario auto administrado, el cual fue asistido por el ordenador (Computer-Assisted Web Interviewing -CAWI), vía online
(Luque, 2017); una técnica que es usada para levantar datos en donde el entrevistado sigue un guion proporcionado en un sitio web mediante un programa para crear entrevistas o cuestionarios web (Szpakowski et al, 2016); mismo que fue habilitado entre el 10 de junio del 2020 y el 25 de junio del mismo año. Esto se debe por la dificultad que se suscitó a nivel mundial para levantar la información en territorio de forma personal, por concepto del confinamiento que ha generado el COVID-19.

En primera instancia, y con el fin de elaborar el cuestionario, se ha 
contemplado tomar en cuenta las 4 fases propuestas por Luque (2017):

1) consideración del contexto de la investigación, 2) la redacción de las preguntas, 3) la estructura, formato y presentación del cuestionario, y 4) la prueba y corrección del cuestionario.

En relación a la primera fase se ha contemplado analizar los componentes del comportamiento del consumidor qué influyen en la lealtad de un individuo sobre los establecimientos gastronómicos del sector de alimentos y bebidas por el impacto que ha generado en esta persona el COVID-19 en el sector de alimentos y bebidas, a partir de la relación existente entre: los estímulos del entorno del consumidor, los estímulos de marketing mix, la actitud del consumidor, y el proceso de decisión de compra del consumidor y la lealtad tal como se describen en las hipótesis previamente formuladas en este estudio.

En cuanto a la redacción y estructuración del cuestionario se consideraron 43 ítems (Ver Tabla 1), los cuales fueron identificados en función de la literatura y ajustados al contexto de esta investigación, que posteriormente fueron agrupados en diferentes dimensiones según cada una de las variables o factores que conforman la lealtad como objeto de estudio.

Para el primer constructo, "Estímulos del entorno (EE)", se agrupó con los siguientes factores: demográfico 3 ítems, tecnológico 2 ítems, sociales 4 ítems, culturales 3 ítems. Para el segundo constructo, "Estímulos de marketing (EM)", se agrupó con los siguientes factores: producto 5 ítems, precio 3 ítems, plaza 6 ítems y promoción 5 ítems. Para el tercer constructo, "Proceso de compra $(P C)$ ", 5 ítems. Finalmente, para el cuarto constructo, "Lealtad (L)", se agrupó con los siguientes factores: cognitivo 3 ítems (Hollander, 1978 referido por Ubillos et al, 2005), afectivo 1 ítem (McGuire, 1968 referido por Ubillos et al, 2005), conativo-conductual 3 ítems (Rosenberg, 1960 y Breckler, 1984 referidos por Ubillos et al, 2005)

Cada uno de estos elementos se midieron a través de escalas Likert de 4 puntuaciones (Maese et al, 2016), los cuales fueron aplicados al cuestionario y que contribuyeron a la comprobación de las hipótesis planteadas. 
Tabla 1. Componentes de la lealtad del consumidor

\begin{tabular}{|c|c|c|}
\hline Variable & Factor & $\begin{array}{l}\text { Dimensiones } \\
\text { (Ítems) }\end{array}$ \\
\hline \multirow{4}{*}{$\begin{array}{l}\text { Estímulos del } \\
\text { entorno } \\
\text { (EE) }\end{array}$} & $\begin{array}{l}\text { Demográfico } \\
\text { (D) }\end{array}$ & $\begin{array}{l}\text { D_1 Edad } \\
\text { D_2 Género } \\
\text { D_3 Ingresos }\end{array}$ \\
\hline & $\begin{array}{l}\text { Tecnológico } \\
\text { (T) }\end{array}$ & $\begin{array}{l}\text { T_1 Accesibilidad } \\
\text { T_2 Conocimiento }\end{array}$ \\
\hline & $\begin{array}{l}\text { Sociales } \\
\text { (S) }\end{array}$ & $\begin{array}{l}\text { S_1 Relaciones sociales } \\
\text { S_2 Relaciones laborales } \\
\text { S_3 Relaciones familiares } \\
\text { S_4 Relaciones de pareja }\end{array}$ \\
\hline & $\begin{array}{l}\text { Culturales } \\
\text { (C) }\end{array}$ & $\begin{array}{l}\text { C_1 Costumbres gastronómicas } \\
\text { C_2 Tradiciones gastronómicas } \\
\text { C_3 Hábitos de consumo }\end{array}$ \\
\hline \multirow{4}{*}{$\begin{array}{l}\text { Estímulos de } \\
\text { marketing } \\
\text { (EM) }\end{array}$} & $\begin{array}{l}\text { Producto } \\
\text { (PRO) }\end{array}$ & $\begin{array}{l}\text { PRO_1 Variedad de productos o servicios } \\
\text { PRO_2 Diseño o características del producto o servicio } \\
\text { PRO_3 Percepción de la marca en cuanto a calidad } \\
\text { PRO_4 Percepción de la marca en cuanto a higiene y } \\
\text { manipulación de los productos } \\
\text { PRO_5 Empaque del producto para servicio a domicilio }\end{array}$ \\
\hline & $\begin{array}{l}\text { Precio } \\
\text { (PRE) }\end{array}$ & $\begin{array}{l}\text { PRE_1 Precio final } \\
\text { PRE_2 Formas de pago } \\
\text { PRE_3 Recargo }\end{array}$ \\
\hline & $\begin{array}{l}\text { Plaza } \\
\text { (PLA) }\end{array}$ & $\begin{array}{l}\text { PRE_1 Canal de distribución } \\
\text { PRE_2 Ubicación } \\
\text { PRE_3 Horario de atención } \\
\text { PRE_4 Proceso de venta } \\
\text { PRE_5 Diseño del establecimiento } \\
\text { PRE_6 Plataformas tecnológicas }\end{array}$ \\
\hline & $\begin{array}{l}\text { Promoción } \\
\text { (PRM) }\end{array}$ & $\begin{array}{l}\text { PRM_1 Publicidad } \\
\text { PRM_2 Venta personal } \\
\text { PRM_3 Promoción de venta } \\
\text { PRM_4 Social media } \\
\text { PRM_5 Relacionas públicas }\end{array}$ \\
\hline $\begin{array}{l}\text { Proceso de } \\
\text { compra } \\
\text { (PC) }\end{array}$ & $\begin{array}{l}\text { Acciones } \\
\text { (A) }\end{array}$ & $\begin{array}{l}\text { A_1 Reconocimiento de las necesidades } \\
\text { A_2 Búsqueda de información } \\
\text { A_3 Evaluación de alternativas } \\
\text { A_4 Decisión de compra } \\
\text { A_5 Comportamiento posterior a la compra }\end{array}$ \\
\hline \multirow{3}{*}{$\begin{array}{l}\text { Lealtad } \\
\text { (L) }\end{array}$} & $\begin{array}{l}\text { Cognitivo } \\
\text { (AC) }\end{array}$ & $\begin{array}{l}\text { AC_1 Creencias hacia un producto o servicio } \\
\text { AC_2 Opiniones hacia un producto o servicio } \\
\text { AC_3 Información hacia un producto o servicio }\end{array}$ \\
\hline & $\begin{array}{l}\text { Afectivo } \\
\text { (AA) }\end{array}$ & $\begin{array}{l}\text { AA_1 Sentimientos de agrado o desagrado hacia un } \\
\text { producto o servicio }\end{array}$ \\
\hline & $\begin{array}{l}\text { Conativo- } \\
\text { conductual } \\
\text { (ACC) }\end{array}$ & $\begin{array}{l}\text { ACC_1 Tendencias de compra ante un producto o servicio } \\
\text { ACC_2 Disposiciones de compra ante un producto o } \\
\text { servicio } \\
\text { ACC_3 Intenciones de compra ante un producto o servicio }\end{array}$ \\
\hline
\end{tabular}

Fuente: Adaptado sobre la base de: Kotler y Keller (2012), Kotler y Armstrong, (2012), Ubillos et al (2005)

Una vez estructurado el formato del

previamente a dos expertos afines al cuestionario, se garantizó su validez tema para su revisión y de contenido; para ello, se envió recomendación previa; mismo que 
contribuyeron posteriormente en el ajuste necesario en cuanto a: instrucciones, redacción, estructura del cuestionario y dificultad de contestar las preguntas.

Consecuentemente, se realizó una prueba piloto a 30 sujetos de estudio (Casas y Repullo, 2003) para identificar si los ítems han sido correctamente comprendidos por todos los sujetos; esto indicará el grado en que todos los elementos de la escala son coherentes entre sí y qué ítems deberían ser eliminados que mejoren la fiabilidad de la escala.

Finalmente, una vez elaborado el cuestionario y realizados los ajustes correspondientes, se levantó nuevamente a 600 encuestas, cifra considerada a partir de una muestra obtenida de una población infinita, con un nivel de significancia del $95 \%$, un error del $4 \%$ y una heterogeneidad del $50 \%$. Entre las personas que colaboraron completando el cuestionario, según datos obtenidos posteriormente al levantamiento, manifiestan que, 274 hombres $(45,7 \%), \quad 324$ mujeres (54\%) y 2 personas que no quisieron identificase en alguno de estos dos géneros. Entre ellos, 169 encuestados $(28,2 \%)$ tienen edades entre 18 y 30 años, 147 (24,5\%) entre 31 a 40 años, 167 (27,8\%) entre 41 y 50 años, y finalmente 117 $(19,5 \%)$ más de 51 años. El equilibrio entre las edades permite disminuir el sesgo durante este estudio.

Para el análisis de las escalas, se procedió en primera instancia un análisis de fiabilidad basado en el cálculo del coeficiente alfa de Cronbach, posteriormente se consideró un análisis factorial exploratorio mediante el cálculo de los coeficientes de correlación, el test estadístico de esfericidad de Bartlett y la medida de adecuación muestral Kaiser-Meyer-Olkin (KMO); finalmente para la comprobación de hipótesis se ha estimado una prueba de chi-cuadrado de independencia, mediante la comparación de la distribución observada de los datos con la distribución esperada de los datos y así poder determinar si el valor observado de una variable depende del valor observado de otra variable. 


\section{Resultados}

\subsection{Análisis de fiabilidad}

Con el fin de llevar a cabo una depuración de las escalas, (a excepción de la escala afectiva del factor lealtad) se realizó un análisis de fiabilidad basado en el cálculo del coeficiente alfa de Cronbach, desde un punto de vista relativo, para realizar una evaluación de consistencia interna. Según Kocak et al (2014) y Belén et al (2015), referidos por Maese et al (2016), y
Luque (2017), consideran que el valor mínimo del coeficiente de fiabilidad debe ser igual o mayor a 0,7; por lo tanto, aquellos ítems que causan conflictos, indican una fiabilidad de consistencia interna no satisfactoria. En relación a la medida de fiabilidad de la escala de medida según alpha de Cronbach, se puede observar en la tabla 2 aquellos ítems que corresponden a las variables: estímulos del entorno, estímulos de marketing, procesos de decisión de compra y lealtad.

Tabla 2. Resultados de análisis de fiabilidad.

\begin{tabular}{|c|c|c|c|}
\hline $\begin{array}{c}\text { Constructo } \\
\text { Factor }\end{array}$ & Alpha de Cronbach & Variables & $\begin{array}{c}\text { Alpha si se elimina el } \\
\text { elemento }\end{array}$ \\
\hline Estímulos del Entorno (EE) & $0,882\left({ }^{\star}\right)$ & & \\
\hline \multirow{3}{*}{ Demográfico (D) } & \multirow{3}{*}{0,719} & D 1 & 0,478 \\
\hline & & $D^{-} 2$ & 0,622 \\
\hline & & D_3 & $0,748\left(^{\star *}\right)$ \\
\hline \multirow{2}{*}{ Tecnológico (T) } & \multirow{2}{*}{0,899} & T_1 & --- \\
\hline & & T_2 & --- \\
\hline \multirow{4}{*}{ Sociales (S) } & \multirow{4}{*}{0,869} & S_1 & 0,816 \\
\hline & & $S_{2} 2$ & 0,824 \\
\hline & & 53 & 0,827 \\
\hline & & $\mathrm{S}_{-}^{-} 4$ & 0,860 \\
\hline \multirow{3}{*}{ Culturales (C) } & \multirow{3}{*}{0,877} & $\mathrm{C}_{-1}^{-1}$ & 0,808 \\
\hline & & C_2 & 0,802 \\
\hline & & C_3 & 0,866 \\
\hline \multirow[t]{2}{*}{ Estímulos de marketing (EM) } & \multicolumn{3}{|l|}{$0,939\left(^{*}\right)$} \\
\hline & \multirow{5}{*}{0,867} & PRO 1 & 0,849 \\
\hline \multirow{4}{*}{ Producto (PRO) } & & PRO_2 & 0,855 \\
\hline & & PRO 3 & 0,820 \\
\hline & & PRO_4 & 0,827 \\
\hline & & PRO 5 & 0,846 \\
\hline \multirow{3}{*}{ Precio (PRE) } & \multirow{3}{*}{0,777} & PRE_1 & 0,683 \\
\hline & & PRE 2 & 0,684 \\
\hline & & PRE_3 & 0,735 \\
\hline \multirow{6}{*}{ Plaza (PLA) } & \multirow{6}{*}{0,815} & PRE 1 & 0,786 \\
\hline & & PRE_2 & 0,776 \\
\hline & & PRE_3 & 0,763 \\
\hline & & PRE_4 & 0,813 \\
\hline & & PRE 5 & 0,784 \\
\hline & & PRE_6 & 0,790 \\
\hline \multirow{2}{*}{ Promoción (PRM) } & \multirow[b]{2}{*}{0,868} & PRM_1 & 0,828 \\
\hline & & PRM 2 & 0,862 \\
\hline
\end{tabular}




\begin{tabular}{|c|c|c|c|}
\hline $\begin{array}{l}\text { Constructo } \\
\text { Factor }\end{array}$ & Alpha de Cronbach & Variables & $\begin{array}{l}\text { Alpha si se elimina el } \\
\text { elemento }\end{array}$ \\
\hline & & $\begin{array}{l}\text { PRM_3 } \\
\text { PRM_4 } \\
\text { PRM_5 }\end{array}$ & $\begin{array}{l}0,845 \\
0,831 \\
0,834\end{array}$ \\
\hline Proceso de compra (PC) & $0,866\left(^{*}\right)$ & & \\
\hline Acciones (A) & 0,866 & $\begin{array}{l}\text { A_1 } \\
\text { A_2 } \\
\text { A_3 } \\
\text { A_4 } \\
\text { A_5 }\end{array}$ & $\begin{array}{l}0,843 \\
0,846 \\
0,820 \\
0,820 \\
0,855\end{array}$ \\
\hline Lealtad (L) & $0,897\left(^{*}\right)$ & & \\
\hline Cognitivo (AC) & 0,859 & $\begin{array}{l}A C \_1 \\
A C \_2 \\
A C \_3\end{array}$ & $\begin{array}{l}0,851 \\
0,780 \\
0,778\end{array}$ \\
\hline Afectivo (AA) & --- & AA_1 & --- \\
\hline Conativo-conductual (ACC) & 0,754 & $\begin{array}{l}\text { ACC_1 } \\
\text { ACC_2 } \\
\text { ACC_3 }\end{array}$ & $\begin{array}{c}0,794\left({ }^{* \star}\right) \\
0,592 \\
0,632\end{array}$ \\
\hline
\end{tabular}

Nota: (*) Escala completa; $\left(^{* *}\right)$ Ítem eliminado para mejorar la fiabilidad de la escala

\subsection{Análisis \\ factorial exploratorio}

Posteriormente, mediante el análisis factorial exploratorio, se permitió conocer la estructura de interrelaciones de las variables (una vez depuradas las variables); de tal forma que en el análisis se utilizarán las puntuaciones de los factores en lugar de las medidas de la variable (Moliner \& Berenguer, 2011). Para ello se consideró el cálculo de los coeficientes de correlación, el test estadístico de esfericidad de Bartlett, con el fin de evaluar la aplicabilidad del análisis factorial de las variables estudiadas, y la medida de adecuación muestral Kaiser-MeyerOlkin (KMO), con el fin de saber si existe correlación entre las variables.
Para este punto se ha estimado trabajar mediante el método de componentes principales y un método de rotación tipo varimax. Los valores que se deben considerar para su aceptación para la validación de escalas de medida se estima que, cuanto más cerca de 1 tenga el valor obtenido del test KMO, implica que la relación entre las variables es alta, $\geq$ 0,9 muy bueno; $\geq 0,7$ buena, $\geq 0,6$; < 0,5 muy bajas; mientras que para la prueba de Barlet debe ser < a 0,05. En la Tabla 3 se puede observar que todos los factores alcanzaron un valor mayor a 0,5 en la medida $\mathrm{KMO}$ y todos obtuvieron un valor menor a 0,05 de significancia según la prueba de Barlet, lo que demuestra medidas satisfactorias. 
Para el análisis factorial, Estímulos del Entorno (EE), (ver Tabla 3) dio como resultado, para el factor demográfico, una sola carga factorial y un $79,87 \%$ de varianza explicada (una vez depurada la variable); lo mismo ocurre con el factor tecnológico $\quad(90,79 \%), \quad$ social $(71,79 \%)$ y cultural $(80,26 \%)$ que mostraron una sola carga factorial. Tanto para el primero y segundo factor (D) y (T), se obtuvieron valores de 0,500 ; lo que implica que la relación entre las variables es muy baja, o por otro lado solo poseen dos dimensiones para que generen valores óptimos.

Tabla 3. Análisis factorial de los Estímulos del Entorno (EE).

\begin{tabular}{|c|c|c|c|c|c|c|}
\hline Factor & Dimensión & $\begin{array}{c}\text { Cargas } \\
\text { del } \\
\text { factor }\end{array}$ & Comunalidades & $\begin{array}{c}\text { \% de } \\
\text { varianza } \\
\text { explicada }\end{array}$ & $\begin{array}{c}\text { Sig. } \\
\text { De } \\
\text { Barlett }\end{array}$ & KMO \\
\hline $\begin{array}{l}\text { Demográfico } \\
\text { (D) }\end{array}$ & $\begin{array}{l}D \_1 \\
D \_2\end{array}$ & $\begin{array}{l}0,894 \\
0,894\end{array}$ & $\begin{array}{l}0,799 \\
0,799\end{array}$ & $79,87 \%$ & 0,000 & 0,500 \\
\hline $\begin{array}{l}\text { Tecnológico } \\
\text { (T) }\end{array}$ & $\begin{array}{l}\text { T_1 } \\
\text { T_2 }\end{array}$ & $\begin{array}{l}0,953 \\
0,953\end{array}$ & $\begin{array}{l}0,908 \\
0,908\end{array}$ & $90,79 \%$ & 0,000 & 0,500 \\
\hline Sociales (S) & $\begin{array}{l}\text { S_1 } \\
\text { S_2 } \\
\text { S_3 } \\
\text { S_4 }\end{array}$ & $\begin{array}{l}0,875 \\
0,864 \\
0,854 \\
0,795\end{array}$ & $\begin{array}{l}0,765 \\
0,746 \\
0,729 \\
0,632\end{array}$ & $71,79 \%$ & 0,000 & 0,748 \\
\hline Culturales (C) & $\begin{array}{l}\text { C_1 } \\
\text { C_2 } \\
\text { C_3 }\end{array}$ & $\begin{array}{l}0,907 \\
0,911 \\
0,869\end{array}$ & $\begin{array}{l}0,823 \\
0,829 \\
0,756\end{array}$ & $80,26 \%$ & 0,000 & 0,732 \\
\hline
\end{tabular}

En cuanto al análisis factorial de los Estímulos de marketing (EM), (Ver Tabla 4) se generó una sola carga factorial en cada uno de los factores contemplados en esta variable y un porcentaje de varianza explicada de $67,06 \%$ para el producto, precio $(69,69 \%)$, plaza $(53,15 \%)$ y promoción de $(65,79 \%)$ respectivamente.

Tabla 4. Análisis factorial de los Estímulos de Marketing (EM)

\begin{tabular}{lcccccc}
\hline \multicolumn{1}{c}{ Factor } & Variables & $\begin{array}{c}\text { Cargas } \\
\text { del } \\
\text { factor }\end{array}$ & Comunalidades & $\begin{array}{c}\text { \% de } \\
\text { varianza } \\
\text { explicada }\end{array}$ & $\begin{array}{c}\text { Sig. De } \\
\text { Barlett }\end{array}$ & KMO \\
\hline & PRO_1 & 0,777 & 0,603 & & & \\
Producto & PRO_2 & 0,750 & 0,562 & & & \\
(PRO) & PRO_3 & 0,888 & 0,788 & $67,06 \%$ & 0,000 & 0,818 \\
& PRO_4 & 0,874 & 0,765 & & & \\
\hline Precio & PRO_5 & 0,797 & 0,635 & & & \\
(PRE) & PRE_1 & 0,845 & 0,714 & & & \\
& PRE_2 & 0,843 & 0,711 & $69,69 \%$ & 0,000 & 0,702 \\
Plaza & PRE_3 & 0,816 & 0,666 & & & \\
(PLA) & PRE_1 & 0,731 & 0,534 & & 0,000 & 0,807 \\
\hline & PRE_2 & 0,746 & 0,557 & $53,15 \%$ & & \\
\hline
\end{tabular}




\begin{tabular}{lllllll}
\hline & PRE_4 & 0,610 & 0,372 & & & \\
& PRE_5 & 0,758 & 0,574 & & & \\
& PRE_6 & 0,712 & 0,508 & & & \\
& PRM_1 & 0,844 & 0,713 & & & \\
Promoción & PRM_2 & 0,741 & 0,549 & & & \\
(PRM) & PRM_3 & 0,791 & 0,626 & $65,79 \%$ & 0,000 & 0,864 \\
& PRM_4 & 0,842 & 0,709 & & & \\
& PRM_5 & 0,832 & 0,693 & & & \\
\hline
\end{tabular}

Con respecto al análisis factorial de los Proceso de compra (PC), (Ver Tabla 5) se generó una sola carga factorial en cada uno de los factores contemplados en esta variable y un porcentaje de varianza explicada de $65,11 \%$ para el factor de acción.

Tabla 5. Análisis factorial de los Proceso de compra (PC)

\begin{tabular}{lcccccc}
\hline Factor & Variables & $\begin{array}{c}\text { Cargas } \\
\text { del } \\
\text { factor }\end{array}$ & Comunalidades & $\begin{array}{c}\% \text { de } \\
\text { varianza } \\
\text { explicada }\end{array}$ & $\begin{array}{c}\text { Sig. } \\
\text { De } \\
\text { Barlett }\end{array}$ & KMO \\
Acciones & A_1 & 0,788 & 0,621 & & & \\
(A) & A_2 & 0,781 & 0,610 & & & \\
& A_3 & 0,856 & 0,732 & $65,118 \%$ & 0,000 & 0,854 \\
& A_4 & 0,858 & 0,736 & & & \\
\hline
\end{tabular}

En cuanto al análisis factorial de los Procesos de compra (PC) (Ver Tabla 6) se generó una sola carga factorial en cada uno de los factores contemplados en esta variable y un porcentaje de varianza explicada del $78,24 \%$ a nivel cognitivo y 82,92 a nivel conativo-conductual de $82.92 \%$ (una vez depurada la variable). no se generaron cargas, ni \% de varianza explicada a nivel afectivo, por poseer una sola dimensión. Por otro lado, las variables ACC2 y ACC_3, obtuvieron valores de 0,500 durante la prueba de $\mathrm{KMO}$, lo que implica que la relación entre las variables es muy baja, o por otro lado solo poseen dos dimensiones para que generen valores óptimos.

Tabla 6. Análisis factorial de la Lealtad (L).

\begin{tabular}{lcccccc}
\multicolumn{1}{c}{ Factor } & Variables & $\begin{array}{c}\text { Cargas } \\
\text { del } \\
\text { factor }\end{array}$ & Comunalidades & $\begin{array}{c}\% \text { de } \\
\text { varianza } \\
\text { explicada }\end{array}$ & $\begin{array}{c}\text { Sig. } \\
\text { De } \\
\text { Barlett }\end{array}$ & KMO \\
\hline $\begin{array}{l}\text { Cognitivo } \\
\text { (AC) }\end{array}$ & AC_1 & 0,854 & 0,729 & & & \\
\hline $\begin{array}{l}\text { Conativo- } \\
\text { conductual }\end{array}$ & AC_2 & 0,899 & 0,808 & $78,24 \%$ & 0,000 & 0,724 \\
(ACC) & ACC_2 & 0,900 & 0,810 & & & \\
\hline
\end{tabular}




\subsection{Comprobación de hipótesis}

En relación con las hipótesis formulas según el modelo propuesto se obtiene que; para la hipótesis Hi1, se obtuvo, mediante prueba Chi cuadrado, un $p$ valor de $0,000<a$ 0,05 y se concluye que existe suficiente evidencia muestral para afirmar que las variables correspondientes a los estímulos del entorno del consumidor sí influyen significativamente en la lealtad. En función a Hi2, también se obtuvo un $p$ valor de $0,000<$ a 0,05 ; por lo tanto, se concluye que los estímulos del marketing mix sí influyen significativamente en la lealtad. Esto mismo sucede con $\mathrm{Hi} 3$ donde se obtuvo, un p valor de 0,000 < a 0,05; se concluye que existe suficiente evidencia muestral para afirmar que el proceso de compra sí influye significativamente en la lealtad.

\section{Conclusiones}

En función a los resultados obtenidos durante este estudio se puede observar que aquellos estímulos que provienen del entorno como aquellos que son generados por las empresas a partir del marketing mix, influyen significativamente en la lealtad hacia una empresa del sector de alimentos y bebidas, lo mismo que sucede con los procesos que se generan durante una compra por parte de un individuo. En este sentido, los antecedentes antes descritos por autores como: Moliner y Berenguer (2011), Ubillos et al (2005), Wu y Li (2018), entre otros, destacan la importancia de varios elementos inherentes para la construcción de lealtad desde su enfoque actitudinal.

Dentro de las variables que conforman a los Estímulos del Entorno, el factor tecnológico es el que genera mayor impacto frente al factor demográfico, social y cultural. Sin lugar a duda, debido a la realidad que vive el mundo y el sector de alimentos y bebidas, y por ello la tendencia que muchos usuarios han adoptado para satisfacer su necesidad de alimentación a través de plataformas tecnológicas. En este sentido dentro de los Estímulos del Marketing el factor precio tiene mayor impacto frente al resto de criterios que conforman al Mix de Marketing; siendo evidente que los ingresos de las familias se han visto afectadas a causa del COVID-19; esto quiere decir que este factor 
guarda una relación importante con

la lealtad del consumidor.

En el análisis factorial de la lealtad, el factor conativo-conductual, la disposición e intención de compra de un producto o un servicio, ejerce mayor impacto frente al factor cognitivo, como las creencias, las opiniones y la información acerca de un producto o un servicio.

Frente a este análisis, existen ciertas implicaciones empresariales que se deben considerar a raíz de este COVID-19; una de ellas es incrementar su oferta gastronómica a medios digitales a fin de que la Lealtad perdure, considerando los datos recopilados en esta investigación, y considerar que los estímulos del entorno y del marketing mix influyen en la decisión de compra del consumidor.

\section{Bibliografía}

Alguacil, M., Dos Santos, M., PastorBarceló, A. y Colino, O. (2016). Análisis cualitativo comparativo de la lealtad en servicios públicos deportivos. Revista de Psicología del Deporte, 25(1), 69-72. https://www.redalyc.org/pdf/2 351/235146293016.pdf
Arguello, S., Arguello, M. y Saltos, W. (2017). La lealtad de los clientes de los hostales de la ciudad de Riobamba Ecuador. Industrial Data, 20(1), 51-57. https://doi.org/10.15381/idata. v20i1.13509

Bietti, L. (2009). Disonancia cognitiva: procesos cognitivos para justificar acciones inmorales. Ciencia Cognitiva: Revista Electrónica de Divulgación, 3(1), 15-17. Obtenido de http://www.cienciacognitiva.or g/files/2009-3.pdf

Bigné, J. y Andreu, L. (2004). Emociones, satisfacción y lealtad del consumidor en entornos comerciales un análisis comparativo entre centro comercial y centro urbano. Distribución y consumo, 14(76), 77-88. https://www.researchgate.net/ profile/Enrique_Bigne2/public ation/28279750_Emociones_ satisfaccion_y_lealtad_del_c onsumidor_en_entornos_com erciales_un_analisis_compar ativo_entre_centro_comercial _y_centro_urbano/links/0c96 053bea8d8576c2000000/Em ociones-satisfaccion-ylealtad-del-consumidor-enentornos-comerciales-unanalisis-comparativo-entrecentro-comercial-y-centrourbano.pdf 
Bitner, M. J. (1991). The evolution of the services marketing mix and its relationship to service quality. En S. Brown, E. Gummesson, B. Edvardsson, \& B. Gustavsson, Quality: A Multidisciplinary and Multinational Perspective (pp. 23-37). New York: Lexington Books.

Booms, B. H. y Bitner, M. J. (1981). Marketing strategies and organization structures for service firms. Marketing of services, 25(3), 47-52.

Candela, A. (2019). Propuesta de implementación de un tablero de control para medir la satisfacción del cliente en un restaurante [Tesis de Grado]. Lima: Universidad San Igancio de Loyola.

Casas, J. y Repullo, J. J. (2003). La encuesta como técnica de investigación. Elaboración de cuestionarios y tratamiento estadístico de los datos (I). Aten Primaria, 31(8), 528538.

www.unidaddocentemfyclasp almas.org.es/resources/9+At en+Primaria+2003.+La+Encu esta+l.+Custionario+y+Estadi stica.pdf

Castro-González, S. y Bande, B. (2016). Influencia de las emociones en la relación entre la Responsabilidad Social Corporativa y la lealtad del consumidor. Esic Market Economics and Business
Journal, $\quad 47(3), \quad 397-421$. https://doi.org/10.7200/esicm. 155.0473.1e

CEPAL. (2 de julio de 2020). Sectores y empresas frente al COVID-19: emergencia y reactivación. Recuperado el 30 de noviembre de 2020, de https://repositorio.cepal.org/bi tstream/handle/11362/45734/ S2000438_es.pdf?sequence $=4 \&$ isAllowed $=\mathrm{y}$

de Almeida, C., da Silva, L., Durieux, F. y Reis, C. (2015). El marketing mix cómo factor de influencia del comportamiento del mercado brasileño para los cruceros marítimos. Turismo y Desarrollo Local, 8(18), 1-21. https://ideas.repec.org/a/erv/t urdes/y2015i1836.html

dos Anjos, C., dos Santos, P., Simões, R. y Mainardes, R. (2019). Definição e implantação de indicadores chave de desempenho (KPI key performance indicator): estudo de caso em uma empresa do ramo fotográfico da cidade de Guarapuava. TCC Administração, 1-21. http://repositorio.camporeal.e du.br/index.php/tccadmin/arti cle/view/297

Draskovic, N. y Valijak A. The 4Cs of the Croatian public healthcare system: social marketing challenges at the dawn of EU accession. World Review of Entrepreneurship, 
Management and Sust. Development, 8(2), 221-235. https://doi.org/10.1504/WRE MSD.2012.046122

Forgas, S., Moliner, M., Sánchez, J. y Palau, R. (2011). La formación de la lealtad de un cliente de una compañía aérea: diferencias entre aerolíneas tradicionales y de bajo coste. Cuadernos de Economía y Dirección de la Empresa, 11, 162-172. https://doi.org/10.1016/j.cede. 2011.02.005

Fornell, C., Johnson, M., Anderson, E., Cha, J. y Bryant, B. (1996). The American Customer Satisfaction Index: Nature, Purpose, and Findings. Journal of Marketing, 60(4), 718.

https://doi.org/10.2307/12518 98

Gallegos, J. (2002). Gestión de alimentos y bebidas para hoteles, bares y restaurantes. Madrid: Paraninfo.

García, C. y Fabero, M. (2016). Desarrollo y validación inicial de una escala para evaluar la fidelidad de los consumidores hacia las tiendas. European Research on Management and Business Economics, 22, 94-100.

https://doi.org/10.1016/j.iedee .2015.10.005

Goi, C. L. (2009). A Review of Marketing Mix: 4 Ps or More?
Internacional Journal of Marketing Studies, 1(1), 2- 15. https://doi.org/10.5539/ijms.v 1n1p2

Gomes, A., De Azevedo, M. y Gomes, A. (2013). El sistema de oferta de restaurantes de alta gastronomía: Una perspectiva sensorial de las experiencias de consumo. Estudios y Perspectivas en Turismo, 22, 336 - 356. https://dialnet.unirioja.es/servl et/articulo?codigo $=5131423$

Hoffman, D. y Bateson, J. (2012). Marketing de servicios: conceptos, estrategias y casos. México D.F.: Cengage Learning.

Huarcaya-Victoria, J. (2020). Consideraciones sobre la salud mental en la pandemia de COVID-19. Revista Peruana de Medicina Experimental y Salud Pública, 37(2), 1-8. doi: https://doi.org/10.17843/rpme sp.2020.372.5419

Hung Do, Q. y Anh Vu, T. (2020). Understanding consumer satisfaction with railway transportation service: An application of 7Ps marketing mix. Management Science Letters, 10(6), 1341-1350. https://doi.org/10.5267/j.msl.2 019.11 .023

Izurieta, M. (2015). Comportamiento de compra de alimentos orgánicos en la ciudad de 
Quito desde la perspectiva del modelo de comportamiento del consumidor final de Philip Kotler. Quito: Universidad Simón Bolívar. Obtenido de http://repositorionew.uasb.ed u.ec/bitstream/10644/4314/1/

PI-2015-12-IzurietaComportamiento\%20de.pdf

Johnson, M., Saletti, L. y Tumas, N. (2020). Emociones, preocupaciones y reflexiones frente a la pandemia del COVID-19 en Argentina. Ciência \& Saúde Coletiva, 4, 1-7.

http://dx.doi.org/10.1590/141 3-81232020256.1.10472020

Kotler, P. y Armstrong, G. (2012). Marketing. Naucalpan de Juárez: Pearson Educación de México, S.A. de C.V.

Kotler, P. y Keller, K. (2012). Dirección de Marketing. México: Pearson Educación.

Lamb, C. (2019). MKTG. Marketing. Ciudad de Mexico: Cenage.

Lombart, C. y Didier, L. (2016). Sources of retailer personality: Private brand perceptions. Journal of Retailing and Consumer Services, 28, 117-125. https://doi.org/10.1016/j.jretco nser.2015.09.002

López, N. (2003). Marketing en restauración. Madrid: Editorial Síntesis.
López, O., Lara, D., Villacis, J., Hernández, H. y Carrión, Á. (2018). El marketing de servicios y su incidencia en la rentabilidad de la Fundación Cultural Ambato. Observatorio de la Economía Latinoamericana, 1-7. https://ideas.repec.org/a/erv/o bserv/y2018i24105.html

Luque, T. (2017). Investigación de marketing 3.0. Madrid: Pirámide.

Macas, M., Bustamante, W. y Romero, M. (2018). ¿La herramienta AGIS contribuye con ISO 9001:2015; a la mejora continua de servicios en las organizaciones? Espacios, 39(33), 17 https://revistaespacios.com/a 18v39n33/18393317.html

Maese, J., Iniesta, A., Valles, D. y Báez, Y. (2016). Coeficiente alfa de Cronbach para medir la fiabilidad de un cuestionario difuso. CULCyT, 13(59), 146156.

Mahajan, P. y Golahit, S. (2017). Incorporating 11 p's of service marketing mix and its impact on the development of technical education. Journal of Entrepreneurship Education, 20(2), 1-16. https://papers.ssrn.com/sol3/ papers.cfm?abstract_id=3101 547

Ministerio de Turismo. (2020). Ministerio de Turismo emite 
protocolo para la reapertura de establecimientos de alimentos y bebidas. Obtenido de https://www.turismo.gob.ec/m inisterio-de-turismo-emiteprotocolo-para-reapertura-deestablecimientos-de-alimen

Moliner, B. y Berenguer, G. (2011). El efecto de la satisfacción del cliente en la lealtad: aplicación en establecimientos minoristas. Cuadernos de Administración, 24(42), 101124.

https://doi.org/10.11144/Javer iana.cao24-42.escl

Munuera, J. y Rodríguez, A. I. (2016). Estrategias de marketing. Un enfoque basado en el proceso de dirección. Madrid: ESIC.

Organización Mundial de la Salud. (2020). COVID 19 e inocuidad de los alimentos: orientaciones para las empresas alimentarias. Obtenido de https://apps.who.int/iris/bitstre am/handle/10665/331856/W HO-2019-nCoV-Food_Safety2020.1-spa.pdf

Osorio, R., Restrepo, L. y Muñoz, H. (2016). Marketing digital: Una mirada al pasado, presente y futuro. Marketing visionario, $5(1)$, 3-20. http://ojs.urbe.edu/index.php/ market/article/view/2386
Panigrahi, A., Aware, K. y Patil, A. (2018). Application of integrated marketing communication in pharmaceutical industry. Journal of Management Research and Analysis, 5(2), 133-139.

https://doi.org/10.18231/2394 $-2770.2018 .0021$

Pappu, R. y Genevieve, P. (2016). How does brand innovativeness affect brand loyalty? European Journal of Marketing, 50(1/2), 1-44. https://doi.org/10.1108/EJM01-2014-0020

Peñalosa, M., Ruiz, G., Juárez, B. y López, D. (2018). Factores de influencia en la lealtad del consumidor de servicios adquiridos a través de internet en México. Caderno Profissional de Marketing Unimep, 6(1), 119-128. https://expeditiorepositorio.ut adeo.edu.co/handle/20.500.1 2010/8705

Rivas, A. e Ildefonso, G. (2013). Comportamiento del consumidor; decisiones y estrategia de marketing. Madrid: ESIC.

Saidani, B. y Sudiarditha, K. (2019). Marketing Mix-7ps: The Effect on Customer Satisfaction. Journal Pendidikan Ekonomi \& Bisnis, 7(7), 72-86. https://doi.org/10.21009/JPE B.007.1.7 
Salazar, D. (2016). Comportamiento del sector de alimentos y bebidas y los elementos del marketing mix en la ciudad de Quito. Turismo y Sociedad, 19, 177-191. http://dx.doi.org/10.18601/01 207555.n19.10

Salazar, D., Quintero, J. y Mena, F. (2016). Criterios de medición en la lealtad del cliente aplicados a restaurantes de la ciudad de Quito. Gran Tour: Revista de Investigaciones Turísticas, 14, 23 - 43. https://eutm.es/grantour/index .php/grantour/article/view/63

Sanz, S., García, C., Rodríguez, J. y Morales, E. (2018). Efecto de la personalidad de tienda y el género del consumidor sobre la lealtad hacia las tiendas. Docfradis, 6, 1-22. https://ideas.repec.org/p/ovr/d ocfra/1806.html

Serra, A. (2012). Marketing turístico. Madrid: Esic Editorial.

Setiawan, Y. (2019). Analysis Retail Marketing Mix and Customer Loyalty. International Journal Management Science \& Business, 1(1), 47-60. https://doi.org/10.17509/msb. v1i1.17364

Sisodiya, P. (2018). The impact of marketing mix model/elements on consumer buying behaviour: a study of fmcg products in Jaipur city. International Journal of
Technical Research \& Science, 3(1), 29-33. https://doi.org/10.30780/IJTR S.V3.11.2018.016

Sohrabi, C., Alsafi, Z., O'Neill, N., Khan, M., Kerwan, A. et al. (2020). World Health Organization declares global emergency: A review of the 2019 novel coronavirus (COVID-19). International Journal of Surgery, 76, 71-76. https://doi.org/10.1016/j.jisu.2 020.02.034

Szpakowski, R., Zając, P., Dykowska, G. et al. (2016). Labour migration of Polish nurses: a questionnaire survey conducted with the Computer Assisted Web Interview technique. Hum Resour Health, 14(24), 80144. https://doi.org/10.1186/s1296 0-016-0124-9

Ubillos, S., Mayordomo, S. y Páez, D. (2005). Actitudes: definición y medición. Componentes de la actitud. Modelo de acción razonada y acción planificada. Psicología Social, Cultura y Educación, 1-24

Uddin, Z. (2016). Using the 7Ps of Booms and Bitner as Controllable Tactical Marketing Tools: An Exploratory Study on Selected Super Shops in Bangladesh. International Journal of Trade \& 
Commerce-IIARTC, 5(1), 1229.

https://www.researchgate.net/ profile/Md_Zahir_Uddin_Arif2 /publication/308075750_Usin g_the_7Ps_of_Booms_and_ Bitner_as_Controllable_Tacti cal_Marketing_Tools_An_Ex ploratory_Study_on_Selected _Super_Shops/links/57d90d9 e08ae0c0081 ef0083/Usingthe-7Ps-of-Booms-andBitner-as-ControllableTactical-Marketing-Tools-AnExploratory-Study-onSelected-SuperShops.pdf\#page $=22$

Wu, Y.-L. y Li, E. (2018). Marketing mix, customer value, and customer loyalty in social commerce: A stimulusorganism-response perspective. Internet Research, 28(1), 74-104. https://doi.org/10.1108/IntR08-2016-0250

Xie, Y. (2020). The relationship among marketing mix, customer satisfaction and customer loyalty of chinese tourists to budget hotel of central Bangkok. UTCC International Journal of Business and Economics, 12(1), 123-142. http://www.ijbejournal.com/im ages/files/21316953225c5d5 9bbe99b4.pdf

Yang, Z. (2020). The relationship among perceived value, customer satisfaction and loyalty of chinese tourist shopping in bangkok: a case of king power downtown complex. UTCC International Journal of Business and Economics, 12(1), 69-86. http://www.ijbejournal.com/im ages/files/19770836755c5d5 c05b0be3.pdf

Zeithaml, V., Bitner, M. y Dayne, G. (2009). Marketing de servicios. México D.F.: McGraw-Hill. 